\title{
Téoros
}

Revue de recherche en tourisme

\section{L’ère des gîtes du passant est enfin arrivée}

\section{Odette Chaput}

Volume 8, numéro 2, juillet 1989

Hébergement et tourisme

URI : https://id.erudit.org/iderudit/1080321ar

DOI : https://doi.org/10.7202/1080321ar

Aller au sommaire du numéro

Éditeur(s)

Université du Québec à Montréal

ISSN

0712-8657 (imprimé)

1923-2705 (numérique)

Découvrir la revue

Citer cet article

Chaput, O. (1989). L’ère des gîtes du passant est enfin arrivée. Téoros, 8(2),

16-18. https://doi.org/10.7202/1080321ar d'utilisation que vous pouvez consulter en ligne.

https://apropos.erudit.org/fr/usagers/politique-dutilisation/ 


\section{L'ère des gîtes du passant est enfin arrivée}

Il y a 25 ans, le rêseau des Agricotours était principalement connu par les fermes de vacances. Aujourd'hui, ce même résèu connaît une popularité grandissante pour le mode d'hébergement les "Gittes du Passant"i. En 1988, on evaluait l"achalandage du réseau à environ 60 000 personnes/nuitede. Plus de 235 résidences réparties à travers 17 régions touristiques du Québec offrent les programmes d'hébergement en Gîtes du Passant cchambre d'hốte et petit dẹjeuner dans une maison privée), Gites â la Ferme (chambre d'hote et pension complète dans une maison de ferme) et Maisons de Campagne (maison de ferme ou chalet tout equipe). Afin de mieux saisir l'évolution d'Agricotours, voici une brève rétrospective de la Fédération.

\section{L'histoire d'Agricotours versus une approche de commerciali- sation}

En 1972-1973, deux resseaux independants de fermes d'hébergement, comme on les appelait a lépoque, sont mis sur pied au Lac St-Jean et dans l'Estrie. Dès le début, le ministère de l'Agriculture, des Pêcheries et de l'Alimentation du Québec (MAPAQ) s'y intéresse, entreprend une étude et publie un rapport intitule "Projet de développement Agro-touristique au Québec". L'objectifétait d'améliorer la situation économique des fermes pour maintenir les gens sur les terres. II faut savoir que les fermes d'hébergement ne sont pas néesd"une demande touristique exprimée mais bien d"un besoin des agriculteurs. Un besoin economique qui s'est wite transformé autrement puisque les pionniers en ont retiré plus de compensations sociales que de dollars. En 1974, trois Agricotours régionaux se forment: Saguenay-Lac St-Jean, Québec et Bas St-Laurent. La même année, I'Union des Producteurs Agricoles (UPA) fait enregistrer les lettres patentes d'une fédération provinciale des Agricotours. De 1974 à 1979, un comité conjoint MAPAQ-UPA est formé et les deux partenaires foumissent l'infrastructure de fonctionnement. A partir de 1979 , le MAPAQ délaisse graduellement le dossier pour le passer au ministère des Loisirs, de la Chasse et de la Pêche (MLCP). Ce demier accorde la première subvention pour l'exercice financier 1980-1981. Au cours de cet exercice, le réseau des Gîtes du Passant a vu le jour dans l'est du Québec, principalement surdes fermes. Après un échec sur la création d'une agence de voyages laissant une perte financière importante, un questionnement profond s'amoree puisque l'hébergement a la ferme ne correspond plus au besoin de la clientèle et la diversité du réseau est trop restreinte. Un ralentissement des activites nous amene en 1984 à une nouvelle approche plus orientée sur le marchéct, par le fait même, a un plan d'orientation fournissant plusieurs éléments pour amorcer un certain virage. De 1984 à 1986, la stratégie utilisée fut celle de restructurer un réseau faible en notoriété et mal positionné dans le marché de l'hébergement en maison privée, Le réseau se retrouvait en phase de lancement. En 1987, une étude de marché confirme que la stratégie devait viser surtout une croissance progressive pour la Fédération des Agricotours, Cette direction stratégique évite ainsi la marginalisation, dans un marché qui risque de voir l'offre augmenter considérablement suite à la Loi 152. Nous savons que la demande québécoise potentielle est denviron 5 a 9 fois plus grande que celle qui est actuellement satisfaite. Apress avoir misé sur des actions de développement beaucoup plus diversifiées et une déréglementation des tarifs, apres avoir développé une panoplie de services aux membres et apres avoir équilibré les sources de revenus de l'organisme et atteint un pourcentage d'autofinancement enviable, la crédibilité de l'organisme est gagnée. Aujourd'hui, la stratégie est orientée vers l'application d'un marketing-mix qui tient compte d'un plande communication et de commercialisation des services. Le fait que le réseau des Agricotours connaisse une notoriété grandissante est que les investissements sont orientés vers une mission axée sur les communications.

Contrairement au début, l'administration et les membres en majorité favorisent une approche de commercialisation qui soit d'entreprise privếe. Cetté attitude est essentielle pour une mise en marché dynamique et soutenue d'un réseau touristique unique en son genre au Québec. La clé du succès est que chaque membre se considère davantage relationniste du réseau et c'est ce qui se produit depuis deux anschez Agricotours.
* Madame Odette Chaput est directrice generale d'Agricotours. 
NOMBRE DE NUITÉES DE 1975 À 1988

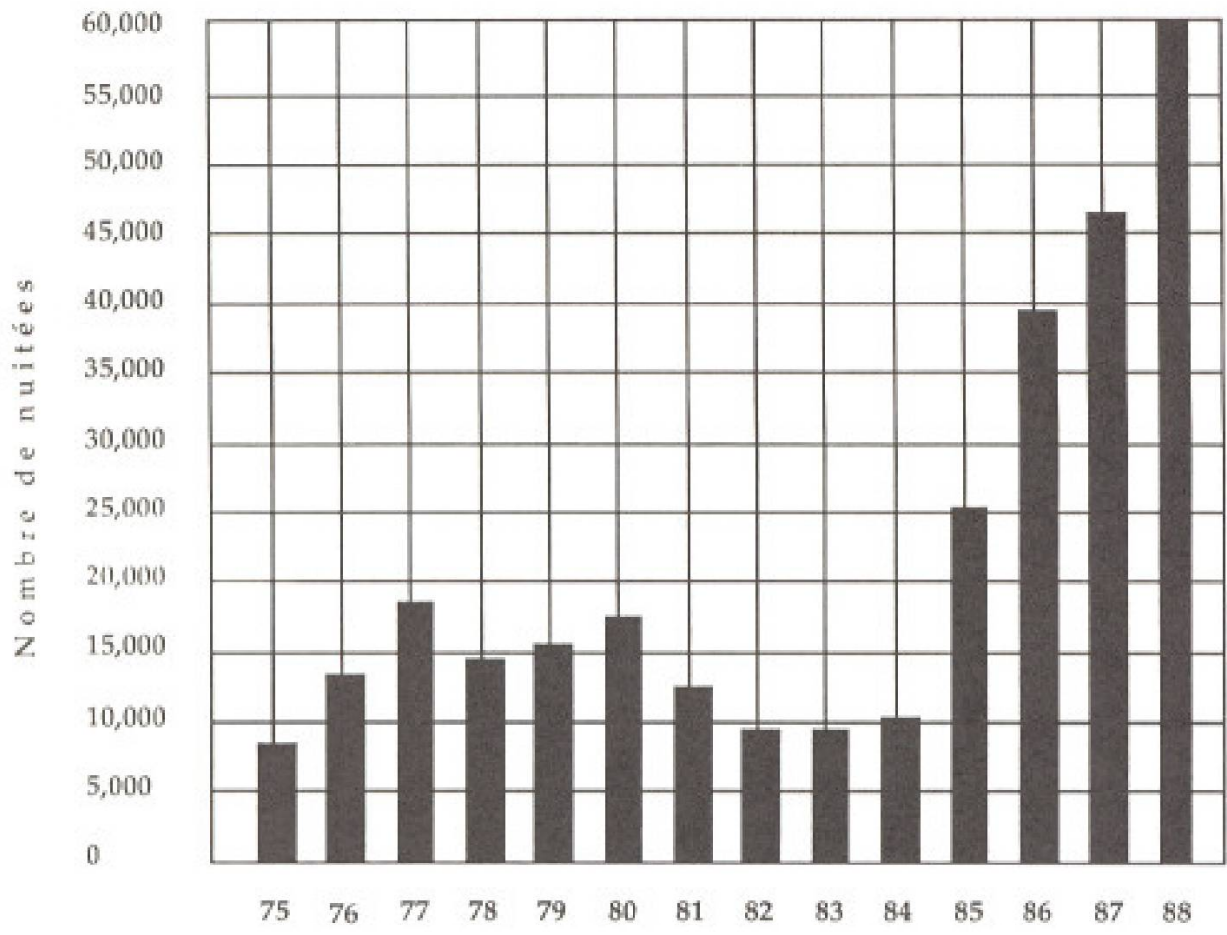

\section{Qualité du réseau}

Le développement d'un réseau d'hébergement qui se veut un développement harmonieux selon l'offre et la demande et surtout de qualité repose, entre autres, sur une politique d'accréditation et un plan de formation. Offrir un service d'hébergement en maisons privées signifie une diversité de l'offre, et, par le fait mème, des normes de qualité souples, Des exigences de base doivent toutefois être présentes: sécurité, hygiène, confort, qualité de nourriture et d'accueil, nombre de chambres, ratio salle de bain/chambre, tarifs. La qualité d'un réseau se retrouve également à travers un bon rapport qualite/prix. Une étude du profil de la clientèle confirme que les principales raisons qui motivent les voyageurs it se rendre dans un Gîte du Passant sont principalement: le type d hébergement familial, son originalité, son hospitalité et l'atmosphère générale ainsi que le prix. De plus, les meilleures cotes de satisfaction aupres de la clientèle sont: la qualité des repas, l'hospitalité et les prix. Plus de $96 \%$ recommanderaient à des parents ou amis de sejoumer dans un Gite du Passant. Il faut en conclure que la formation doit demeurer une priorité au sein du développement car la clientèle semble satisfaite du concept en général. L'avantage qu'aura la Fédération en 1990, c'est de travailler plus à la formation aupres de ses membres plutôt qu'au recrutement car, à présent, les demandes d'adhésion au réseau parviennent régulièrement étant donné sa populari-

\section{La clientèle du réseau Agrico- tours}

Compte tenu que parmi les 3 programmes d'hebergement celui des Gîtes du Passant est le plus étendu, les données qui suivent représentent principallement ce progranme. Une étude du profil de la clientèle dénontre que les gens dont l'äge se situe entre 29 ct 39 ans sont très présents et que le pourcentage de la clientềle âgée de 55 ans et plus augmente considérablement d'année en année. On constate que la majorité des gens sont mariés ou vivent avec un conjoint et qu'une forte majorite des gens n'ont pas d'enfant. Le revenu moyen des répondants se situe entre 30 $000 \$$ et 49 999\$. On retrouve en majorité des gens avec un niveau de scolarité élevé (baccalauréat), suivis par les diplômés du niveau secondaire. On remarque, au niveau de l'occupation des utilisateurs, que les gens sont semiprofessionnels, spécialisés et non spécialisés.

On peut même avancet qu'un certain nombre d'entre eux travaillent en cducation ou en relations humaines. Ils proviennent principalement du Montréal-Métropolitain. Ce profil devrait suivre la même tendance pour 1988. En 1987, on cstimait qu'environ $60 \%$ de la clientèle etait québécoise et que $40 \%$ provenait de l'extérieur du Québec, dont $20 \%$ d'Europe.

Une autre caractéristique est qu'ils voyagent plus que l'ensemble des voyageurs, leurs voyages d'importance s'effectuant le plus souvent is l'extérieur du Québec. Ils affectionnent la vie de groupe et particulièrement le raffinement. La clientèle actuelle sait que le Gîte du Passant est un type dhébergement personnalisé, sympathique et chaleureux, qu'il favorise la connaissance de la culture et du milieu de la région visitéc, qu'il favorise le contact humain, l'échange avec les gens, que le coût est raisonnable et que le service est adéquat. tion d'un comité portant sur l'analyse des commentaires et plaintes des clients, élaboration d'une procédure de traitement de plaintes. A l'heure actuelle, suivant l'application de la Loi 152, la Fẻdération travaille à la rểalisation dun code d'êthique destine à ses membres.

En 1988-1989, les actions de développement ont generé les tésultats apparaissant aux titbleaux suivants:

\begin{tabular}{|c|c|c|}
\hline Année & Demande d'information & Demande de visite \\
\hline $1989^{*}$ & $627^{*}$ & 110 \\
\hline 1988 & 378 & 103 \\
\hline 1987 & 275 & 78 \\
\hline 1986 & 171 & 57 \\
\hline Annce & Membership & $\begin{array}{c}\text { \% augmentation/ } \\
\text { diminution }\end{array}$ \\
\hline $1989^{\circ}$ & $255^{*}$ & $18 \%$ \\
\hline 1988 & 213 & $45 \%$ \\
\hline 1987 & 147 & $17 \%$ \\
\hline 1986 & 126 & $(-14 \%)$ \\
\hline 1985 & 146 & $\cdots$ \\
\hline
\end{tabular}

17 TÉoros - Val. 7, na 5, juillet 1909 


\section{Conclusion}

La Fédération a franchi plusieurs étapes dans son cheminement depuis sa fondation, et, pour 1990 , son 15 e anniversaire d'existence représente une phase de réussite dans l'évolution d'un réseau d'accueil en maison privée de 235 gittes pour une capacité daccueil de 710 chambres. La concurrence est presente et chacun essaie d'atteindre de nouveaux créneaux en diversifiant l'offre de service, soit: chambre avee salle de bain privée et petit salon, service de déjeuner au lit, brunch disponible sur place, gite offrant des concerts, expositions d'art, forfaits avec theâtres d'été, centre d'équitation, centre de ski, pèche, croisière, etc... Pour être compétitif, la Fédération a dû́ adopter de nouvelles politiques de développement et l'extension des tarifs maximum imposés par la Fédération à ses membres. a doté le réseau des gites de nouvelles résidences. offrant plus de services, jouissant d'une situation privilégice ou situés dans une région où le prix du marché est plus élevé. En 1989, le réseau possède beaucoup plus de gites adaptés aux besoins de la clientêle. Parmi les nouveaux développements, la stratégie de communication à venir au niveau de l'image institutionnelle est celle d'établir la notoriété du "réseau des Gites du Passant" comme étant le réseau d'accueil reconnu et apprécié de ce genre d'hébergement au Québec et garantissant une qualité. Au niveau du produit, il sagit de faire connaitre l'existence du Gite du Passant en l'inserant davantage dans les choix d'hébergement touristique. Actuellement, la Fédération diversifie plus que jamais ses activites promotionnelles et voit a Telaboration d'une strategie de commercialisation de ses produits. Paralleclement, une étude d'un plan de financement est en cours, car, on le sait, la problématique des petits organismes, c'est le manque de ressources humaines et financières. Lobjectif à atteindre d'ici quelques années est darriver à une indépendance financière. Parmi les organismes oeuvrant dans le secteur tourisme, la Fédération est un des organismes qui a le plus évolué financièrement. En 1986, on parlait de $20 \%$ d'autofinancement, en 1989 , les êtats financiers démontrent $51 \%$. Agricotours. c'est une association cn plein essor, résolument tournếe vers l'avenir, forte de son expérience dans le domaine et s'appuyant sur un plan d'action orienté vers les besoins du marehé. Bien sûr, la réussite dépend de la volonté des 255 membres à travailler ensemble, ce qui fait la force du réseau, de leur dynamisme en tant qu'ambassadeurs du tourisme au Québec, de leur confiance envers la Fédération pour l'avancement des dossiers et finalement, de leur perseverance à rendre visible un réseau qui se voulait marginal mais qui, grâce à l'effor de chacun, atteint un virage important dans son développement.
ÉVOLUTION DU MEMBERSHIP DE 1975 À 1989

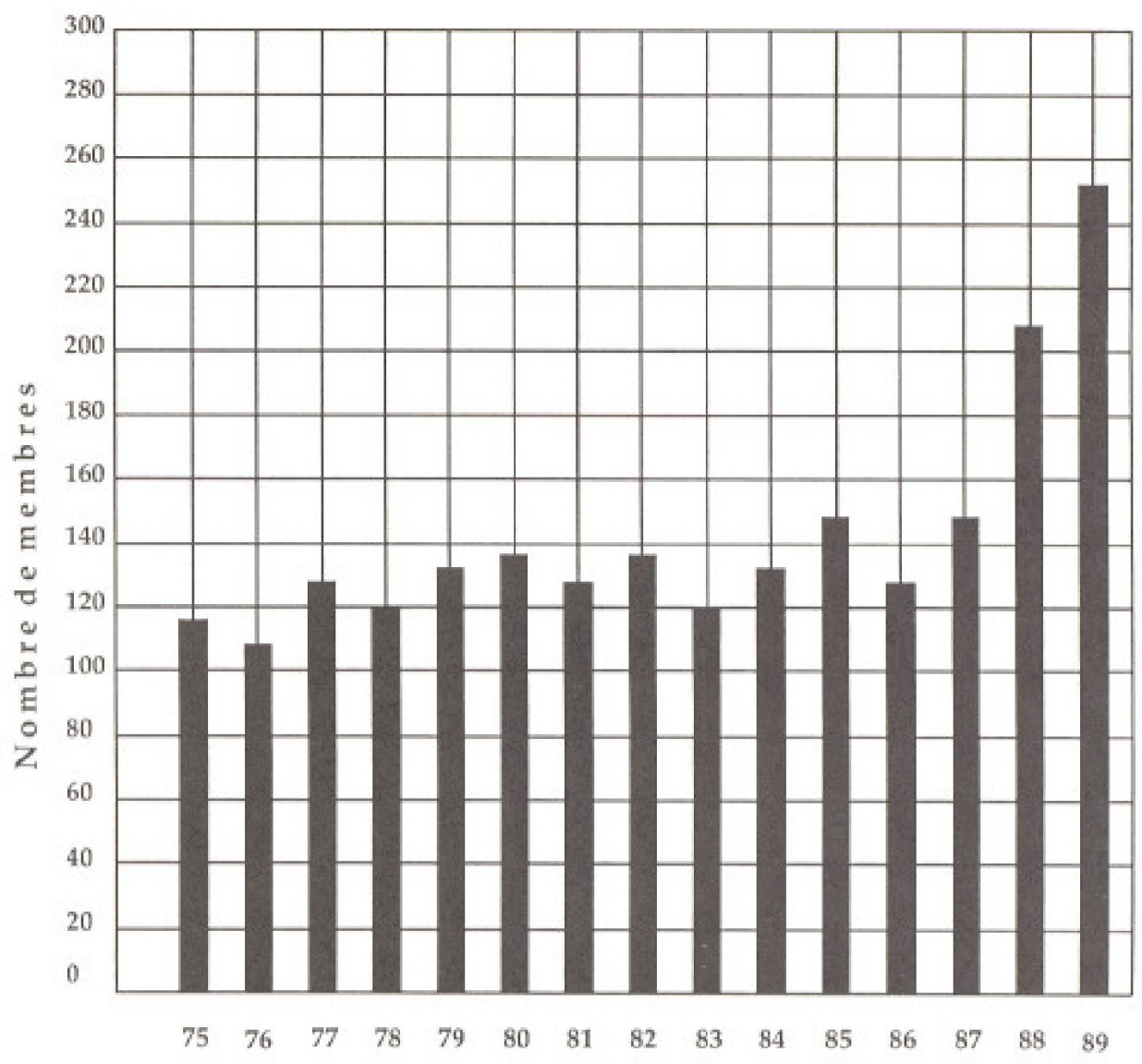

\section{NOTE EXPLICATIVE}

(1) Marque de commerce deposies per la Federation des Agricotours du Qubbec. 